\title{
Graph coloring using commuting order product prime graph
}

\author{
Muhammed Bello ${ }^{a, *}$, Nor Muhainiah Mohd Alib, Surajo Ibrahim Isah ${ }^{b}$ \\ ${ }^{a}$ Department of Mathematics and Computer Science, Federal University of Kashere, P.M.B 0182 Gombe, Gombe State, Nigeria. \\ ${ }^{b}$ Department of Mathematical Sciences, Faculty of Science, Universiti Teknologi Malaysia, 81310 UTM Johor, Bahru, Johor, Malaysia.
}

\begin{abstract}
The concept of graph coloring has become a very active field of research that enhances many practical applications and theoretical challenges. Various methods have been applied in carrying out this study. Let $G$ be a finite group. In this paper, we introduce a new graph of groups, which is a commuting order product prime graph of finite groups as a graph having the elements of $\mathrm{G}$ as its vertices and two vertices are adjacent if and only if they commute and the product of their order is a prime power. This is an extension of the study for order product prime graph of finite groups. The graph's general presentations on dihedral groups, generalized quaternion groups, quasi-dihedral groups, and cyclic groups have been obtained in this paper. Moreover, the commuting order product prime graph on these groups has been classified as connected, complete, regular, or planar. These results are used in studying various and recently introduced chromatic numbers of graphs.
\end{abstract}

Keywords: Commuting order product prime graph, order product prime graph, chromatic number, graph coloring.

2020 MSC: 05C25, 20F65.

(C)2021 All rights reserved.

\section{Introduction}

The concept of defining graphs of groups is very important in algebra, because it creates a bridge to move from group theory to graph theory by defining graphs of groups and investigate the geometric properties of the groups. This study has been established in [8] by Cayley, when he defined the graph that explains the abstract structure of groups generated by set of generators. As a result, many graphs of groups came into existence, for instance, non-commuting graph was defined by Newmann in [18] which is the complement of commuting graph. Later in [7], Bertram used the combinatorial properties of the commuting graph to prove three fundamental and non-trivial theorems on finite groups. Recently, Rajkumar and Devi in [19], introduced intersection graph of cyclic subgroups of finite groups and classify finite groups whose intersection graphs of cyclic subgroups is one of totally disconnected, complete, star, path, cycle. Beside these graphs, power graph is another graph of groups that was introduced in [14] as a tool to studying the combinatorial properties of groups with infinite sequence. The strong metric dimension of this graph on finite groups was characterized in [15]. In addition, Chattopadhyay et al. in

\footnotetext{
${ }^{*}$ Corresponding author

Email addresses: mbello@fukashere.edu.ng (Muhammed Bello), normuhaini@utm.my (Nor Muhainiah Mohd Ali), i isah@gmail. com (Surajo Ibrahim Isah)
}

doi: $10.22436 /$ jmcs.023.02.08

Received: 2020-03-08 Revised: 2020-08-07 Accepted: 2020-08-18 
[10] determined the exact value for the connectivity of the power graph on finite cyclic groups. Bello et al. define order product prime graph of finite groups in [6], as a graph having the elements of a group as its vertices and any two vertices are adjacent if and only if the product of their order is a prime power. This graph started attracting the attention of researchers, for instance in [5], the topological indices of the graph are explored.

On the other hand, the concept of graph coloring originates from coloring the countries of a map such that each face have literal color. The concept have been growing and extend to many practical situations and theoretical challenges making it to become very active field of research. For instance, in compiler optimization, the register allocation uses graph coloring techniques for assigning large number of target program into a small number of CPU registers. Many coloring methods exist and much recently, some new coloring methods are introduced which include dominator coloring and locator coloring. The concept of dominator coloring was introduced by Gera et al. in [12]. A graph has a dominator coloring if it has a proper coloring in which each vertex of the graph dominates every vertex of the same color class and the dominator chromatic number $\chi_{d}(\Gamma)$ is the minimum number of color class in a dominator coloring of the graph $\Gamma$ [12]. Motivated by [12], Arumugam et al. [1] extend this study by obtaining several results on graph coloring and relates to dominator chromatic number and vertex chromatic number or domination number. For more on dominator chromatic number, see [16, 17, 21].

The concept of locating coloring was established by Chatrand et al. in [9]. They consider the connected graph and establish some bounds for the locating chromatic number of the connected graph. This graph coloring also attracted the attention of researchers, for instance, Behtoei and Omoomi [4] investigated the locating chromatic number of grids, the Cartesian product of paths, complete graphs and cartesian product of two complete graphs. Recently, Furuya and Matsumoto [11] obtained the sharp upper bounds of the locating chromatic number of trees by using the number of leaves. For more researches on the locating chromatic number, see [2,3,13].

In this paper, we extend the work of Bello et al. in [6], by introducing commuting order product prime graph of finite groups, as a graph having the elements of a group as its vertices, and two vertices $x, y$ are adjacent if and only if $\mathrm{O}(x) \mathrm{O}(y)=p^{s}, s>0$ and $x y=y x$. We then investigate the general presentations of the graph on cyclic groups, dihedral groups, generalized quaternion groups and quasidihedral groups. Moreover, we classified groups whose commuting order product prime graph is one of connected, complete, regular or planar. Furthermore, motivated by [9, 12], we used the commuting order product prime graph in studying various and recently introduced chromatic numbers, which cover various classes of graphs with significant that have not been covered so far.

\section{Notations and preliminaries}

In this section, we give some basic concepts, notations and preliminaries useful to this paper.

All groups considered in this paper are finite and the investigation covered all finite groups and more specifically on cyclic groups $\mathbb{Z}_{n}=<g>\ni g \in \mathbb{Z}_{n}$, dihedral groups $D_{n}=\left\{a, b \mid a^{n}=b^{2}=(a b)^{2}=e\right\}$, generalized quaternion groups $\mathrm{Q}_{4 \mathrm{n}}=\left\langle\mathrm{a}, \mathrm{b} \mid \mathrm{a}^{2 \mathrm{n}}=e, \mathrm{~b}^{2}=\mathrm{a}^{\mathrm{n}}, \mathrm{bab^{-1 }}=\mathrm{a}^{-1}\right\rangle$ and quasi-dihedral groups

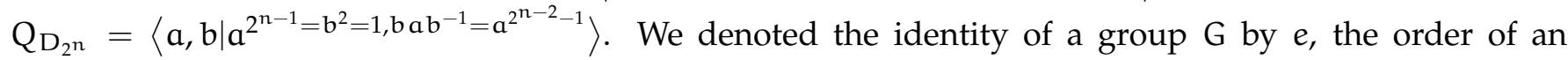
element $a$, by $O(a)$, the centralizer of an element $a \in G$ by $C_{G}(a)=\{y \in G \mid a y=y a\}$, the number of elements in a given sets $A$ by $|A|$.

On the other hand, we consider simple undirected graphs without loop or multiple edges. The sets of vertices and edges of a graph $\Gamma$ are denoted by $V(\Gamma)$ and $E(\Gamma)$, respectively. We denote the adjacency of vertices $v_{1}, v_{2}$ by $v_{1} \sim v_{2}$, number of vertices of the graph $\Gamma$ by $|\mathrm{V}(\Gamma)|$, the degree of the vertex $v$ by $\operatorname{deg}(v)$, the distance between the vertices $v_{1}$ and $v_{2}$ by $\mathrm{d}\left(v_{1}, v_{2}\right)$ which is defined as the number of edges in the shortest path connecting them. A graph $\Gamma$ is regular if all the vertices of the graph have the same degree, that is if for all vertices $v_{1}, v_{2}, \ldots, v_{n}$ of $\Gamma, \operatorname{deg}\left(v_{1}\right)=\operatorname{deg}\left(v_{2}\right)=\cdots=\operatorname{deg}\left(v_{n}\right)$ and a graph $\Gamma$ is $r$-regular if $\operatorname{deg}\left(v_{i}\right)=r, i \in \mathbb{N}$. A graph $\Gamma$ is connected if there is a path between every pair of its vertices, and a graph is complete if there is an edge between every pair of its vertices. A graph is planar if it can be 
drawn in a plane without edge crossing, the neighborhood of a vertex $x \in \mathrm{V}(\Gamma)$ with radius 1 is denoted by $S_{1}(x)=\{a \in \Gamma: d(a, x) \leqslant 1\}$. Throughout this paper $p$ denotes prime number, and $s \in \mathbb{N}$.

The results in Corollary 2.1 and Theorem 2.2, give the description for the order of elements of cyclic groups.

Corollary 2.1 ([20]). In a finite cyclic group, the order of an element divides the order of the group.

Theorem 2.2 ([20]). If $\mathrm{d}$ is a positive divisor of $\mathrm{n}$, the number of elements of order $\mathrm{d}$ in a cyclic group of order $\mathrm{d}$ is $\phi(d)$.

Theorem 2.3 ([6]). Let $\mathrm{G}$ be a cyclic group, $\mathbb{Z}_{\mathrm{n}}$, then

$$
\Gamma^{o p p}(G)= \begin{cases}K_{p^{\alpha}}, & \text { if } n=p^{\alpha}, \\ K_{1}+\bigcup_{i=1}^{d} K_{\left(p_{i}^{\alpha_{i}}-1\right)} \cup \bar{K}_{n+d-\left(1+\sum_{i=1}^{d} p_{i}^{\alpha_{i}}\right),} \text { if } n=\prod_{i=1}^{d} p_{i}^{\alpha_{i}},\end{cases}
$$

where $\mathrm{d}$ is the number of prime divisors of $\mathrm{n}$ and $\alpha \in \mathbb{N}$.

\section{Results and discussion}

In this section, we give the formal definition of the commuting order product prime graph in which an example that visualized the definition follows.

Definition 3.1 (Commuting order product prime graph). Let $\mathrm{G}$ be a finite group, the commuting order product prime graph of $G$, is a graph $\Gamma^{\operatorname{copp}}(\mathrm{G})$, whose vertices are the elements of $G$ and two vertices $u, v$ are adjacent if and only if $\mathrm{O}(u) \mathrm{O}(v)=\mathrm{p}^{s}$ and $u v=v u$, where $s \in \mathbb{N}$ and $p$ a prime number.

Example 3.2. Let $\mathrm{G}=\mathrm{Q}_{\mathrm{D}_{8}}$, then $\mathrm{C}_{\mathrm{G}}(\mathrm{a})=\mathrm{C}_{\mathrm{G}}\left(\mathrm{a}^{3}\right)=\left\{e, \mathrm{a}, \mathrm{a}^{2}, \mathrm{a}^{3}\right\}, \mathrm{C}_{\mathrm{G}}\left(\mathrm{a}^{2}\right)=\mathrm{C}_{\mathrm{G}}(\mathrm{e})=\mathrm{G}$ and $\mathrm{C}_{\mathrm{G}}(\mathrm{b})=$ $\left\{e, b, a^{2} b\right\}, C_{G}(a b)=\left\{e, a b, a^{3} b\right\}$. The commuting order product prime graph of $G$ is given in Figure 1.

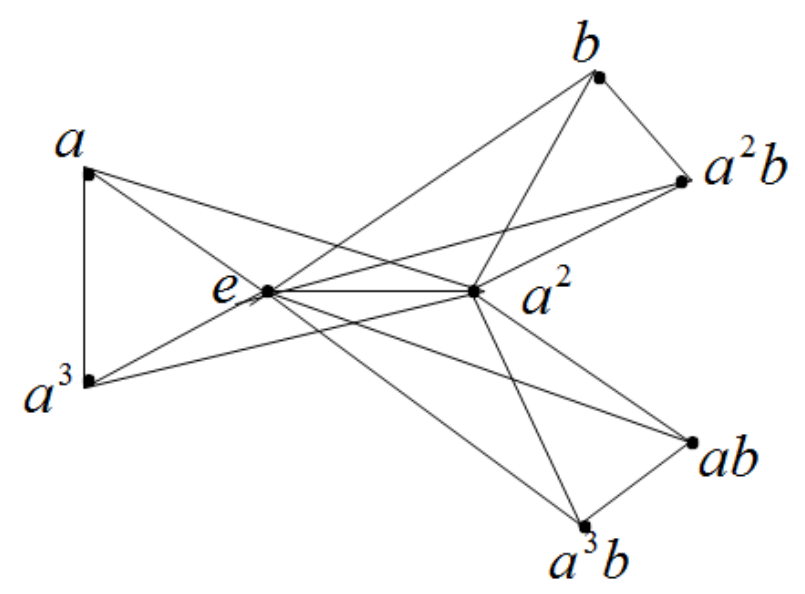

Figure 1: Commuting order product prime graph of $\mathrm{Q}_{\mathrm{D}_{4}}$.

Some results on the vertex degrees of the commuting order product prime graph on some groups are given here, which will be used in investigating other properties of the graph.

Proposition 3.3. Let $G$ be a finite cyclic p-group, then $\operatorname{deg}_{\mathrm{G}}(\mathrm{a})=|\mathrm{G}|-1, \forall \mathrm{a} \in \mathrm{G}$.

Proof. $\mathrm{G}$ is abelian as a p-group and as a result of that, for each $a \in G, O(a)$ is a prime power, so $\operatorname{deg}_{\mathrm{G}}(\mathrm{a})=\left|\mathrm{C}_{\mathrm{G}}(\mathrm{a})\right|-1=|\mathrm{G}|-1$. 
Proposition 3.4. Let $\mathrm{G}$ be a finite cyclic group of composite order, then

1. $\operatorname{deg}(a)=\sum \phi\left(p^{s}\right)$, where $O(a)=p^{k}, 1 \leqslant k \leqslant s ;$

2. $\operatorname{deg}(a)=0$, if $O(a)=\prod p_{i}^{s_{i}}$;

3. $\operatorname{deg}(e)=\sum \phi\left(p_{i}^{s_{i}}\right)$.

Proof. Let $A_{i}=\left\{a \in G \mid O(a)=p_{i}^{k}\right\}, B=\left\{b \in G \mid O(b)=\prod p_{i}^{k}\right\}$. By Theorem 2.2, $\left|A_{i}\right|=\sum \phi\left(p_{i}^{k_{i}}\right)$.

1. Let $a \in G \ni O(a)=p^{k}$, then by Corollary 2.1, $p^{k} /|G|$. Now, pick arbitrary $a_{1} \in A_{1}, a_{2} \in A_{2}$, then $a_{1} \nsim a_{2}$. Thus, $A_{i}$ are distinct cliques of sizes $\phi\left(p_{i}^{k_{i}}\right)$, for each $i$, but $a_{1} \sim e \sim a_{2}$. Therefore $\operatorname{deg}(a)=\sum \phi\left(p^{k_{i}}\right)$.

2. If $O(a)$ is composite then $a$ is isolated vertex by definition and $\operatorname{sodeg}(a)=0$.

3. Observe that $G$ can be partitioned into $G=\{e\} \cup A_{i} \cup B$. By definition, $B$ contains isolated vertices. Pick arbitrary, $a_{i} \in A_{i}$, then $a_{i} \sim e$ for all $i$. Therefore, $\operatorname{deg}(e)=\left|\left\{e \cup A_{i}\right\}\right|-1=\left|A_{i}\right|=\sum \phi\left(p_{i}^{s_{i}}\right)$.

The general presentation of the commuting order product prime graph on cyclic group is given in Theorem 3.5.

Theorem 3.5. Let $\mathrm{G}$ be a cyclic group, $\mathbb{Z}_{\mathrm{n}}$. Then $\Gamma^{\mathrm{copp}}(\mathrm{G}) \cong \Gamma^{\mathrm{opp}}(\mathrm{G})$.

Proof. Let $v_{1}, v_{2} \in \mathrm{V}\left(\Gamma^{\operatorname{copp}}(\mathrm{G})\right)$, then $v_{1} \sim v_{2}$ if $\mathrm{O}\left(v_{1}\right) \mathrm{O}\left(v_{2}\right)=\mathrm{p}^{\mathrm{s}}, \mathrm{s} \in \mathbb{N}$ and if $v_{1} v_{2}=v_{2} v_{1}$. The second condition is trivial since $G$ is abelian and the first condition is the condition for the vertex adjacency of $\Gamma^{\mathrm{opp}}(\mathrm{G})$. Hence $\Gamma^{\mathrm{copp}}(\mathrm{G}) \cong \Gamma^{\mathrm{opp}}(\mathrm{G})$.

The vertex degrees of the elements of dihedral groups, quasi-dihedral groups and generalized quaternion groups are given in Proposition 3.6.

Proposition 3.6. Let $\mathrm{G}$ be one of the groups, $\mathrm{D}_{n}, \mathrm{Q}_{\mathrm{D}_{2 n}}$ or $\mathrm{Q}_{4 n}$, then

(i) $Z(G)=\left\langle a^{\frac{|G|}{4}}\right\rangle$;

(ii) $\langle a\rangle \cong \mathbb{Z}_{n, i}$

(iii) $C_{G}\left(a^{i} b\right)=\left\{\begin{array}{ll}\left\{e, a^{\frac{|G|}{4}}, a^{i} b, a^{i+\frac{|G|}{4}} b\right\}, & \text { if } Z(G) \neq e, \\ \left\{e, a^{i} b\right\}, & \text { if } Z(G)=e,\end{array} ;\right.$

(iv) $\operatorname{deg}\left(a^{i} b\right)= \begin{cases}3, & \text { if } Z(G) \neq e, \\ 1, & \text { if } Z(G)=e ;\end{cases}$

(v) $\operatorname{deg}\left(a^{i}\right)= \begin{cases}\sum_{|G|} \phi\left(p^{s_{i}}\right), & \text { if } \mathrm{O}(a)=p^{s}, \\ \frac{|G|}{2}-1, & \text { if } \mathrm{G} \text { is } p \text {-group, } \\ 0, & \text { if } \mathrm{O}(\mathrm{a})=\prod p_{i}^{s_{i}} ;\end{cases}$

(vi) $\operatorname{deg}(\mathrm{Z}(\mathrm{G}))=\frac{|\mathrm{G}|}{2}+\sum \phi\left(2^{s_{i}}\right) \ni 2^{s_{i}}=\mathrm{O}(\mathrm{a})$;

(vii) $\operatorname{deg}(e)=\frac{|G|}{2}+\sum \phi\left(p_{i}^{s_{i}}\right) \ni p_{i}^{s_{i}}=O(a)$.

Proof. The proofs of (i) and (ii) are straightforward, so we ignore them. To prove (iii), we consider when $n$ is even or odd. Suppose $n$ is even then from (i) above, $Z(G)=\left\langle a^{\left\{\frac{|G|}{4}\right\}}\right\rangle$ and so $C_{G}\left(a^{i} b\right)=\left\langle\left\{a^{i} b, a^{\frac{|G|}{4}}\right\}\right\rangle=$ $\left\{e, a^{\left\{\frac{|G|}{4}\right\}}, a^{i} b, a^{\left\{i+\frac{|G|}{4}\right\}} b\right\}$. But if $n$ is odd then $Z(G)$ is trivial, so in this case $C_{G}\left(a^{i} b\right)=\left\langle a^{i} b\right\rangle=\left\{e, a^{i} b\right\}$. Thus, $\operatorname{deg}\left(a^{i} b\right)=1$. To prove of (iv), the order of each element in $C_{G}\left(a^{i} b\right)$ is 2 and so satisfied the first condition to be clique, so we next check the commutativity status among them. Now from (iii) of this proposition, $\left|\left(C_{G}\left(a^{i} b\right)\right)\right|$ is 4 if $Z(G)$ is non-trivial and 2 otherwise. Therefore, $\operatorname{deg}\left(a^{i} b\right)=\left|C_{G}\left(a^{i} b\right)\right|-1$, so, the, $\operatorname{deg}\left(a^{i} b\right)=3$ if $Z(G) \neq e$ and 1 if $Z(G)$ is trivial. To prove (v), if $O(a)=p^{s}$, then by Theorem 2.2 , there exist $\phi\left(p^{s}\right)$ elements of such order. But such prime can exist with distinct powers, whereas for all distinct powers, a particular prime form single clique, hence $\operatorname{deg}_{G}(a)=\sum \phi\left(p^{s_{i}}\right)$. We move to the situation when $G$ is a p-group. If $G$ is p-group, then $|G|$ must be even prime power. Let $R$ be the set of 
rotations of $G$, then $a^{i} \in R$ and $R \cong \mathbb{Z}_{\frac{|G|}{2}}$. Thus, $R$ is abelian and since $G$ is $p$-group, then $R$ is a clique of size $|R|-1=\frac{|G|}{2}-1$. The case when $O(a)=\prod p_{i}^{s_{i}}$ is proved in Proposition 3.4. For the prove of (vi), since $O(Z(G))=2$, then all $a^{i} b \sim Z(G) \sim a$, where $O(a)=2^{s_{i}}$. Therefore $\operatorname{deg}(Z(G))=\frac{|G|}{2}+\sum \phi\left(2^{s_{i}}\right)$. Finally, to prove (vii), for all $a \in G \ni O(a)=p_{i}^{s_{i}}$, then $a \sim e \sim a^{i} b$, therefore $\operatorname{deg}(e)=\frac{|G|}{2}+\sum \phi\left(p_{i}^{s_{i}}\right)$.

Before we give the general presentation for the commuting order product prime graph of the dihedral groups, generalized quaternion groups and quasi-dihedral groups, Lemma 3.7 is needed as the supporting result that helps in determining the nature and the number of commuting elements in the group.

Lemma 3.7. Let $\mathrm{G}$ be a dihedral group, $\mathrm{D}_{n}, \mathrm{n}$ even, then $\left(\mathrm{a}^{\mathrm{i}} \mathrm{b}, \mathrm{a}^{\frac{|\mathrm{G}|}{4}+\mathrm{i}} \mathrm{b}\right), \mathrm{i}=0,1,2, \ldots, \frac{|\mathrm{G}|}{4}-1$ commute, where $\mathrm{a}$ and $\mathrm{b}$ are the rotations and the reflections of $\mathrm{G}$.

Proof. It follows from (iii) of Proposition 3.6 that if $Z(G)$ is nontrivial, then $a^{i} b$ belong to the center of $\left(a^{\frac{|G|}{4}+i} b\right)$ and so they therefore commute for all $i$.

The general presentation of the commuting order product prime graph on dihedral groups is given in Theorems 3.8 and 3.9, respectively.

Theorem 3.8. Let $\mathrm{G}$ be a dihedral group, $\mathrm{D}_{\mathrm{n}}, \mathrm{n}=\mathrm{p}^{\mathrm{s}}, \mathrm{s} \in \mathbb{N}$, for some prime $\mathrm{p}$, then

$$
\Gamma^{\operatorname{copp}}(G)= \begin{cases}2 K_{1}+\left(K_{n-2} \cup \frac{n}{2} K_{2}\right), & \text { if } p=2, \\ K_{1}+\left(K_{n-1} \cup \bar{K}_{n}\right), & \text { if } p \neq 2 .\end{cases}
$$

Proof. Observe that $\{R \cup e\} \triangleleft G$. If $p=2$, then $Z(G) \subset R$ and therefore $(R-Z(G))$ is a clique of size $n-2$. Since $Z(G)$ is non-trivial, then by Lemma 3.7, $\frac{n}{2}$ pairs of the elements of $F$ commute. Observe that $r \nsim f \forall r \in R, f \in F$, where $r \neq Z(G)$, but $r \sim\{e, Z(G)\} \sim f$. Therefore $\Gamma^{c o p p}(G)=2 K_{1}+\left(K_{n-2} \cup \frac{n}{2} K_{2}\right)$. If $p \neq 2$, then $R$ is a clique of size $n-1$. Now all the elements of $F$ are isolated vertices of the graph since $Z(G)$ is trivial. Pick $r \in R$ and $f \in F$, then $r \nsim f$, but $r \sim e \sim f$. Therefore, $\Gamma \operatorname{copp}(G)=K_{1}+\left(K_{n-1} \cup \bar{K}_{n}\right)$.

Theorem 3.9. Let $\mathrm{G}$ be a dihedral group, $\mathrm{D}_{\mathrm{n}}, \mathrm{n}=\prod_{i=1}^{\mathrm{d}} \mathrm{p}_{i}^{s_{i}}, \mathrm{~s} \in \mathbb{N}$, for some prime $\mathrm{p}$, then

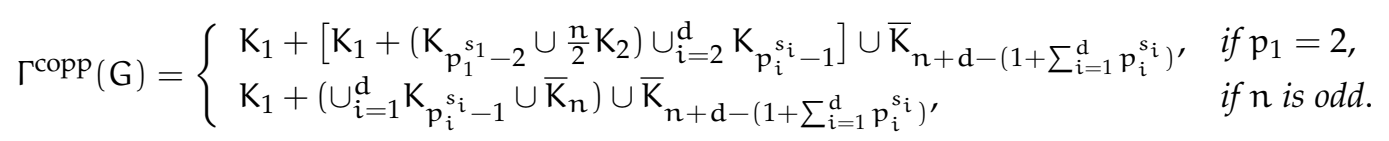

Proof. Since $n=\prod_{i=1}^{d} p_{i}^{s_{i}}$, then each element of order $p_{i}^{s_{i}}$ belongs to $R$, therefore each element of order $p^{s^{\prime}}$ where $s^{\prime}$ is the greatest power of the prime $p_{i}$, generates a cyclic subgroup of size $\left(p^{s^{\prime}}-1\right)$. But $\{R \cup e\} \triangleleft G$, so these cyclic subgroups are cliques in the graph, that is there is total number of $d$ such cliques $C_{i}$. Let $I$ be the set of isolated vertices of the graph then $I \subset R$ and

$$
|\mathrm{I}|=|\mathrm{R}|-\left(\sum_{i=1}^{\mathrm{d}}\left(\mathrm{p}_{\mathrm{i}}^{\mathrm{s}_{\mathrm{i}}}-1\right)\right)=(\mathrm{n}-1)-\mathrm{p}_{1}^{\mathrm{s}_{1}}-\mathrm{p}_{2}^{\mathrm{s}_{2}}-\cdots-\mathrm{p}_{\mathrm{i}}^{\mathrm{s}_{\mathrm{i}}}+\mathrm{d}=\mathrm{n}+\mathrm{d}-\left(1+\sum_{i=1}^{\mathrm{d}} \mathrm{p}_{\mathrm{i}}^{\mathrm{s}_{\mathrm{i}}}\right) .
$$

If $p_{1}=2$, then $n$ is even and $Z(G)$ is non-trivial. By Lemma 3.7, there exist $\frac{n}{2}$ commuting pairs in $F$, let $F^{\prime}$ be the set of these commuting pairs. Pick $x_{1}, x_{2} \in C_{i}, f \in F^{\prime} \ni O\left(x_{1}\right)=2^{s_{1}}, O\left(x_{i}\right)=p^{s_{i}}, O(f)=2,2 \leqslant i \leqslant d$ and $x_{1} \neq Z(G)$. Then $\left\{x_{1}, x_{i}, f\right\}$ is an independent set since $x_{1} \nsim x_{i} \nsim f$, but $x_{1} \sim\{e, Z(G)\} \sim f$ and $x_{i} \sim e$. Therefore,

$$
\begin{aligned}
\Gamma^{\operatorname{copp}}(\mathrm{G}) & =K_{1}+\left[K_{1}+\left(K_{2} s_{1}-2 \cup \frac{n}{2} K_{2}\right) \cup_{i=2}^{d} K_{p_{i}}^{s_{i}}\right] \cup \bar{K}_{I} \\
& =K_{1}+\left[K_{1}+\left(K_{p_{1}^{s_{1}}-2} \cup \frac{n}{2} K_{2}\right) \cup_{i=2}^{d} K_{p_{i}}^{s_{i}}\right] \cup \bar{K}_{n+d-\left(1+\sum_{i=1}^{d} p_{i}^{s_{i}}\right)} .
\end{aligned}
$$

If $n$ is odd, then $p_{i} \neq 2, \forall i \in \mathbb{N}$ and therefore $Z(G)$ is trivial, so set $F$ is an independent set in the graph. 
Let $f \in F, x_{i} \in C_{i}$, then $x_{i} \nsim f$, but $x_{i} \sim e \sim f$. Therefore

$$
\Gamma^{\operatorname{copp}}(\mathrm{G})=\mathrm{K}_{1}+\left(\cup_{i=1}^{\mathrm{d}} \mathrm{K}_{p_{i}^{s_{i}}} \cup \bar{K}_{n}\right) \cup \bar{K}_{I}=K_{1}+\left(\cup_{i=1}^{d} K_{p_{i}^{s_{i}}} \cup \bar{K}_{n}\right) \cup \bar{K}_{n+d-\left(1+\sum_{i=1}^{d} p_{i}^{s_{i}}\right)} .
$$

The characterization of the commuting order product prime graph on dihedral groups in terms of its connectivity, completeness, regularity and planarity for all degrees is given in Proposition 3.10.

Proposition 3.10. Let $\mathrm{G}$ be a dihedral group, $\mathrm{D}_{\mathrm{n}}$. Then $\Gamma^{\mathrm{copp}}(\mathrm{G})$ is connected if $\mathrm{n}=\mathrm{p}^{\mathrm{s}}$, not complete, not regular and planar if $\mathrm{p}_{i}^{s_{i}}<5$, where $\mathrm{p}$ is a prime $\mathrm{p}$ number.

Proof. If $n=p^{s}$, then $R$ and $F$ are cliques, even though $F$ is not clique if $p \neq 2$, but $r \sim e \sim f, \forall r \in R, f \in F$, hence each vertex of the graph is reachable through e. Therefore $\Gamma^{\operatorname{copp}}(\mathrm{G})$ is connected.

On the other hand, if $n=\prod_{i=1}^{d} p_{i}^{s_{i}}$, then there exist $h \in G \ni h \prod_{i=1}^{d} p_{i}^{s_{i}}=e$, such elements are isolated vertices which are not reachable from others, hence the graph is not connected in this case.

Now we can see that for $r \in R, f \in F, r \nsim f$ by the vertex adjacency of $\Gamma^{\operatorname{copp}}(G)$, therefore $\Gamma^{\operatorname{copp}}(G)$ is not complete. It has been seen from the above that for $r \neq Z(G), r \nsim f$ but $r \sim e \sim f$, hence $\operatorname{deg}(e)>$ $\operatorname{deg}(r) \geqslant \operatorname{deg}(f)$, showing that the graph is also not regular since the degree of the vertices varies.

By Theorems 3.8 and 3.9, the maximum complete component of the graph is $p_{i}^{s_{i}}$, therefore $\Gamma^{\operatorname{copp}}(\mathrm{G})$ is planar if $p_{i}^{s_{i}}<5$.

Some chromatic numbers of the commuting order product prime graph on dihedral groups are given here. The investigation begin with the vertex chromatic number, which is given in Proposition 3.11.

Proposition 3.11. Let $\mathrm{G}$ be a dihedral group, $\mathrm{D}_{\mathrm{n}}$. Then

$$
\chi\left(\Gamma^{\operatorname{copp}}(\mathrm{G})\right)= \begin{cases}\mathrm{n}, & \text { if } \mathrm{n}=\mathrm{p}^{\mathrm{s}}, \\ \left|\max \left(\mathrm{p}_{\mathrm{i}}^{\mathrm{s}_{i}}\right)\right|, & \text { otherwise. }\end{cases}
$$

Proof. Let $M$ be set of the maximum complete subgraph of $\Gamma^{\operatorname{copp}}(\mathrm{G})$, then we need at least $|M|$ distinct colors for proper vertex coloring. Let $N_{i}$ and $N_{j}$ be any two disjoint components of $\Gamma^{\operatorname{copp}}(G)$, and pick arbitrary $n_{1} \in N_{i}, n_{2} \in N_{j}$, then $n_{1} \nsim n_{2}$. Meaning that $n_{1}$ and $n_{2}$ can be assigned with same color. Thus, the minimum color needed to color $V\left(\Gamma^{\operatorname{copp}}(G)\right)$ properly is $|M|$. By Theorem 3.9, $|M|=n$ if $n=p^{s}$ and otherwise $\left|\max \left(p_{i}^{s_{i}}\right)\right|$. Therefore, $\chi\left(\left(\Gamma^{\operatorname{copp}}(G)\right)=n\right.$ if $n=p^{s}$ and $\chi\left(\left(\Gamma^{\operatorname{copp}}(G)\right)=\left|\max \left(p_{i}^{s_{i}}\right)\right|\right.$ otherwise.

The dominated chromatic number of the commuting order product prime graph on dihedral groups is given in Proposition 3.12.

Proposition 3.12. Let $\mathrm{G}$ be a dihedral group, $\mathrm{D}_{\mathrm{n}}$. Then

$$
\chi_{\operatorname{dom}}\left(\Gamma^{\operatorname{copp}}(\mathrm{G})\right)= \begin{cases}\mathrm{n}, & \text { if } \mathrm{n}=\mathrm{p}^{\mathrm{s}}, \\ \omega\left(\Gamma^{\mathrm{copp}}(\mathrm{G})\right)+|\mathrm{I}|, & \text { otherwise, }\end{cases}
$$

where $\mathrm{I}$ is the set of the isolated vertices.

Proof. If $n=p^{s}$, then by Proposition 3.11, $\mathrm{V}\left(\Gamma^{\operatorname{copp}}(\mathrm{G})\right)$ requires $n$ distinct colors for proper coloring, we need $n$ color classes. By Theorem 3.8, $\Gamma^{\operatorname{copp}}(G)$ has two central vertices. Let $C_{i}$ for $1 \leqslant i \leqslant n$ be the color classes of $V\left(\Gamma^{\operatorname{copp}}(G)\right)$ and pick $x_{i} \in C_{i}$, then to each color class $C$ of the non-central vertices, we have that $d(e, C)=d(Z(G), C)=1$. Thus, each non-central vertex is dominated by the central vertices and vice-versa. Therefore, each color class $C_{i}$ is dominated by at least one vertex. Hence, $\chi_{\operatorname{dom}}\left(\Gamma^{\operatorname{copp}}(G)\right)=n$. Suppose $n \neq p^{s}$, then by Theorem 3.9, there exist some isolated vertices which must be assigned with distinct colors (to be singleton classes). Also all the non-isolated vertices of the graph are adjacent to central vertex (vertex of $\mathrm{K}_{1}$ ), which dominated their color classes. By Theorem 3.9, the minimum color required to color the graph is $\max \left(p_{i}^{s_{i}}\right)$. Therefore, $\chi_{\operatorname{dom}}\left(\Gamma^{\operatorname{copp}}(G)\right)=\max \left(p_{i}^{s_{i}}\right)+|I|=\omega\left(\Gamma^{\operatorname{copp}}(G)\right)+$ $|\mathrm{I}|$.

The Locating chromatic number of the order product prime graph on dihedral groups is given in Proposition 3.13. 
Proposition 3.13. Let $\mathrm{G}$ be a dihedral group, $\mathrm{D}_{\mathrm{n}}, \mathrm{n}=\mathrm{p}^{\mathrm{s}}, \mathrm{s} \in \mathbb{N}$. Then

$$
\chi_{\mathrm{L}}\left(\Gamma^{\mathrm{copp}}(\mathrm{G})\right)= \begin{cases}\mathrm{n}+2, & \text { if } \mathrm{p}=2, \\ \mathrm{n}+1, & \text { otherwise. }\end{cases}
$$

Proof. Let $R=\left\{e, a, a^{2}, \ldots, a^{n-1}\right\}$ and $F=\left\{b, a b, \ldots, a^{n-1} b\right\}$ be the sets of the rotations and the reflections of $G$, then $R$ is a clique of size $n$. Let $C_{i}$ be the color classes of $V\left(\Gamma^{\operatorname{copp}}(G)\right)$ and define the distance $d\left(x, C_{i}\right)=\min \left\{d(x, a) \mid a \in C_{i}\right\}$. To each vertex $v$, we can define a color code for $v$ as $C_{\pi}(v)=\left(d\left(v, C_{1}\right), d\left(v, C_{2}\right), \ldots, d\left(v, C_{k}\right)\right)$. If $p=2$, then by Lemma 3.7, $\frac{n}{2}$ pairs of elements of $F$, that is $\left(a^{i} b, a^{\frac{n}{2}+1} b\right)$, for $0 \leqslant i \leqslant \frac{n}{2}-1$ are cliques. Then each element of set $R$ shall be given distinct color class. To utilize the minimum number of color class, we shall share some of the color classes of the maximum clique, which is $\mathrm{R}$ with some elements of $\mathrm{F}$ since they are distinct cliques, such that for any two vertices, $v_{i}, v_{j} \in \mathrm{V}\left(\Gamma^{\operatorname{copp}}(\mathrm{G})\right)$, we have that $C_{\pi}\left(v_{i}\right) \neq C_{\pi}\left(v_{j}\right)$. Now, for any $a_{i} \in R$ and $a^{i} b \in F$, we have that $C_{\pi}\left(a_{i}\right) \neq C_{\pi}\left(a^{i} b\right)$, since they belong to distinct cliques. Therefore, the color codes are as follows; $C_{1}=\{e\}, C_{2}=\left\{a^{\frac{n}{2}}\right\}, C_{3}=\{a, b\}, C_{4}=\left\{a^{2}, a b\right\}, \ldots, C_{\frac{n}{2}+1}=\left\{a^{\frac{n}{2}-1}, a^{\frac{n}{2}-2} b\right\}, C_{\frac{n}{2}+2}=\left\{a^{\frac{n}{2}+1}, a^{\frac{n}{2}-1} b\right\}, C_{\frac{n}{2}+3}=$ $\left\{a^{\frac{n}{2}+2}, a^{\frac{n}{2}} b\right\}, \ldots, C_{n}=\left\{a^{n-1}, a^{n-3} b\right\}, C_{n+1}=\left\{a^{n-2} b\right\}, C_{n+2}=\left\{a^{n-1} b\right\}$. Hence, $\chi_{L}\left(\Gamma^{\operatorname{copp}}(G)\right)=n+2$. If $p \neq 2$, then $R$ is a clique and $F$ is an independent set, so the class colors of $R /\{e\}$ can be shared with some elements of $F$. Now $|R /\{e\}|=|F|-1$, so we need one additional color class for an element of $F$. Therefore, the color classes are $C_{1}=\{e\}, C_{2}=\left\{a^{j}, a^{j-1} b\right\}$ for $2 \leqslant i \leqslant n+1,1 \leqslant j \leqslant n-1$. Hence, $\chi_{\mathrm{L}}\left(\Gamma^{\operatorname{copp}}(\mathrm{G})\right)=\mathrm{n}+1$.

The investigation of the edge chromatic number of the commuting order product prime graph on dihedral groups is given in Proposition 3.14 and Proposition 3.15, respectively.

Proposition 3.14. Let $\mathrm{G}$ be a dihedral group, $\mathrm{D}_{\mathrm{n}}$, where $\mathrm{n}=\mathrm{p}^{\mathrm{s}}, \mathrm{s} \in \mathbb{N}$. Then $\chi^{\prime}\left(\Gamma^{\operatorname{copp}}(\mathrm{G})\right)=2 \mathrm{n}-1$.

Proof. Let $R$ and $F$ be the set of the non-trivial rotations and the reflections of $G$, then $A$ is a clique. If $p=2$, then by Theorem 3.8, the vertices $\left\{e, a^{\frac{n}{2}}\right\}$ of $2 K_{1}$ are central. Thus, there exist edges from all the vertices of the graph to $e$ and $a^{\frac{n}{2}}$. That is $\operatorname{deg}(e)=\operatorname{deg}\left(a^{\frac{n}{2}}\right)$ is the maximum degree. As a result, we need at least $\operatorname{deg}(e)=2 n-1$ colors to properly color the edges incident to the central vertex $e$. But the edges incident to $e$ are different from those incident to $a^{\frac{n}{2}}$. Therefore the colors for the edges incident to $e$ are sufficient to color the edges incident to $a^{\frac{n}{2}}$ and also to color the remaining edges of the graph. Hence, $\chi^{\prime}\left(\Gamma^{\operatorname{copp}}(G)\right)=2 n-1$. If $p \neq 2$, then $Z(G)$ is trivial and so $R=\left\{b, a b, a^{2} b, \ldots, a^{n-1} b\right\}$, is an independent set. Thus, $a^{i} b \sim e \sim a^{j}$, for all $0 \leqslant i \leqslant n-1,1 \leqslant j \leqslant n-1$. Therefore $\operatorname{deg}(e)=|R|+|F|=2 n-1$, but since $\mathrm{R}$ is a clique, then the colors for the edges of the vertices of $\mathrm{R}$ that are incident to $e$ is sufficient to color the edges adjacent to the entire elements of $R$. Thus, we need $|R|+|F|$ colors to properly color the entire edges of the graph. Therefore, $\chi^{\prime}\left(\Gamma^{\operatorname{copp}}(G)\right)=2 n-1$.

Proposition 3.15. Let $\mathrm{G}$ be a dihedral group, $\mathrm{D}_{\mathrm{n}}$, where $\mathrm{n}=\prod p_{i}^{s_{i}}, s_{i} \in \mathbb{N}$. Then $\chi^{\prime}\left(\Gamma^{\mathrm{copp}}(\mathrm{G})\right)=\mathrm{n}+$ $\sum_{i=1}^{d}\left(p_{i}^{s_{i}}-1\right)$.

Proof. Let $R$ and $F$ be the sets of the rotations and the reflections of $G$, respectively and let $A_{i}=\{x \in$ $\left.R \mid O(x)=p_{i}^{s_{i}}, s_{i}>0\right\}$. Since $n=\prod p_{i}^{s_{i}}$, then $|G|=2 \prod p_{i}^{s_{i}}$. Thus, there are some elements $h \in G$ such that $\mathrm{O}(\mathrm{h})=2 \prod p_{i}^{k_{i}}, k \in \mathbb{N}$, which can not be considered for this coloring since they are edge-less. Suppose $p_{1}=2$ and let $B=\left\{a^{i} b, a^{i+\frac{n}{2}} b\right\}$, where $a^{i} b, a^{i+\frac{n}{2}} b \in F$. Then corresponding to each prime $p_{i}$ in $A_{i}$, there is a clique of size $\phi\left(p_{i}^{s_{i}}\right)$. Let $A^{\prime}$ be the set of these cliques, also in set $B$, corresponding to each $i$, there is a clique of size two. Now, for any vertex $x \in A^{\prime}$ and $y \in F$, we have that $x \sim e \sim y$, thus $\operatorname{deg}(e)=\left|A^{\prime}\right|+|F|=n+\sum_{i=1}^{d}\left(p_{i}^{s_{i}}-1\right)$ is the maximum degree. Thus, we need at least $n+\sum_{i=1}^{d}\left(p_{i}^{s_{i}}-1\right)$ colors to properly color the edges of the graph. It is easy to see that these colors are sufficient to color the entire edges of the graph, since by the vertex adjacency, $\left\{e, a^{\frac{n}{2}}, a^{i} b, a^{i+\frac{n}{2}} b\right\}$ is a clique where two edges of $a^{i} b, a^{i+\frac{n}{2} b}$ are incident to $e$ and also to $a^{\frac{n}{2}}$. Thus, two colors can be managed by $a^{i} b, a^{i+\frac{n}{2} b}$ for $a$ particular $i$. Therefore, $n+\sum_{i=1}^{d}\left(p_{i}^{s_{i}}-1\right)$ colors can be utilized for proper coloring of the edges of the 
graph. Thus, $\chi^{\prime}\left(\Gamma^{\operatorname{copp}}(G)\right)=n+\sum_{i=1}^{d}\left(p_{i}^{s_{i}}-1\right)$. If $p_{i} \neq 2$, then $Z(G)$ is trivial and so $F$ is an independent set. Pick arbitrary $x_{i} \in A_{i}$ and $y \in F$, then $x_{i} \sim e \sim y$, therefore $\operatorname{deg}(e)=\left|A_{i}\right|+|F|=\sum_{i=1}^{d} \phi\left(A_{i}\right)+n=$ $n+\sum_{i=1}^{d}\left(p_{i}^{s_{i}}-1\right)$. Now since $|B|>\left|A_{i}\right|$, then the edges of the vertices of set $B$ that are adjacent to $e$ are more than that of set $A_{i}$ that are adjacent to $e$. Therefore the colors for the edges incident to the central vertex $e$ are sufficient to color the edges of the entire graph. Thus, $\chi^{\prime}\left(\Gamma^{\operatorname{copp}}(G)\right)=n+\sum_{i=1}^{d}\left(p_{i}^{s_{i}}-1\right)$.

The vertex and edge coloring of the commuting order product prime graph on dihedral group of degree five is given in Figure 2.

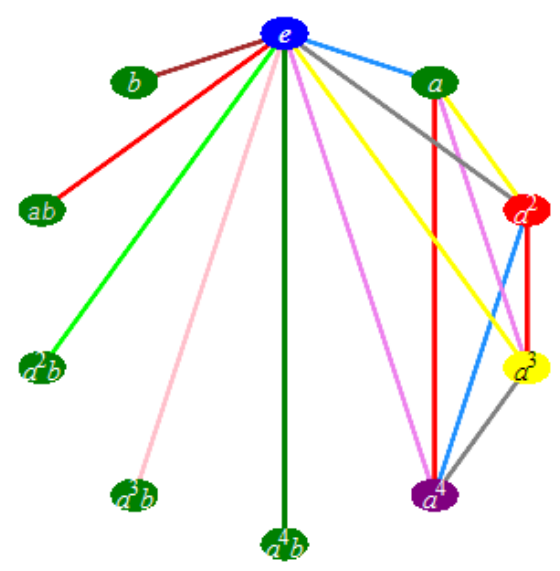

Figure 2: Vertex and edge coloring of the commuting order product prime graph of the dihedral group of degree five.

The results on the commuting order product prime graph on generalized quaternion group are given here. The investigation begins with the structure for the graph, which is given in Theorems 3.16 and 3.17, respectively.

Theorem 3.16. Let $\mathrm{G}$ be a generalized quaternion group, $\mathrm{Q}_{4 \mathrm{n}}, \mathrm{n}=\mathrm{p}^{\mathrm{s}}, \mathrm{s} \in \mathbb{N}$. Then

$$
\Gamma^{\operatorname{copp}}(\mathrm{G})= \begin{cases}2 \mathrm{~K}_{1}+\left(\mathrm{K}_{2(n-1)} \cup n \mathrm{~K}_{2}\right), & \text { if } \mathrm{p}=2, \\ \mathrm{~K}_{1}+\left[\left(\mathrm{K}_{1}+n \mathrm{~K}_{2}\right) \cup \mathrm{K}_{n-1}\right] \cup \bar{K}_{n-1}, & \text { otherwise. }\end{cases}
$$

Proof. We proof the Theorem by considering the group presentation. Let $H=\left\langle a^{i}\right\rangle$ for $0 \leqslant i \leqslant 2 n-1$, then $[H, G]=2$. Thus, $H \triangleleft G$. Therefore, all $a^{i}$ satisfy the first condition to be adjacent to any other vertex. On the other hand, the centralizer, $C_{G}\left(a^{i} b\right)=\left\langle a^{i} b, a^{n}\right\rangle$, for $0 \leqslant i \leqslant 2 n-1$, so each $\left\{a^{i} b, a^{i+n} b\right\}, 0 \leqslant i \leqslant$ $2 n-1$, is a clique of size 2 . Now, we need to count the number of elements in $H$ and $C_{G}\left(a^{i} b\right)$, we can see that $|H|=2 n$ and $\left|C_{G}\left(a^{i} b\right)\right|=4$. If $p=2$, then $e \sim\left(a^{i}, a^{i} b\right) \sim Z(G)$, for all $i$. Therefore, $H /\{e, Z(G)\}$ and $C_{G}\left(a^{i} b\right) /\{e, Z(G)\}$ are cliques of sizes $2 n-2$ and 2 , respectively. Hence,

$$
\Gamma^{\operatorname{copp}}(\mathrm{G})=2 \mathrm{~K}_{1}+\left(\mathrm{K}_{|\mathrm{H} /\{\mathrm{e}, \mathrm{Z}(\mathrm{G})\}|} \bigcup n \mathrm{~K}_{\mid \mathrm{C}_{\mathrm{G}}\left(\mathrm{a}^{\mathrm{i}} \mathrm{b} /\{\mathrm{e}, \mathrm{Z}(\mathrm{G})\} \mid\right.}\right)=2 \mathrm{~K}_{1}+\left(\mathrm{K}_{2(\mathrm{n}-1)} \cup \mathrm{nK} \mathrm{K}_{2}\right) .
$$

If $p \neq 2$, then we have the following sets; $N=\left\{x \in G \mid O(x)=p^{t}, 1 \leqslant t \leqslant s\right\}$ and $M=\{y \in G \mid O(y)=$ $\left.4 \mathrm{p}^{\mathrm{t}}, 1 \leqslant \mathrm{t} \leqslant \mathrm{s}\right\}$, where $|\mathrm{N}|=\phi\left(\mathrm{p}^{\mathrm{s}}\right)=|\mathrm{M}|=\mathrm{n}-1$. By the vertex adjacency, $\mathrm{x} \sim \mathrm{e}$, but $\mathrm{M}$ is a set of isolated vertices. Therefore,

$$
\Gamma^{\operatorname{copp}}(\mathrm{G})=\mathrm{K}_{|e|}+\left[\left(\mathrm{K}_{|\mathrm{Z}(\mathrm{G})|}+\mathrm{nK}_{\left|\mathrm{C}_{\mathrm{G}}\left(\mathrm{a}^{\mathrm{i}} \mathrm{b}\right) /\{\mathrm{e}, \mathrm{Z}(\mathrm{G})\}\right|}\right) \cup \mathrm{K}_{|\mathrm{N}|}\right] \cup \overline{\mathrm{K}}_{|\mathrm{N}|}=\mathrm{K}_{1}+\left[\left(\mathrm{K}_{1}+n \mathrm{~K}_{2}\right) \cup \mathrm{K}_{\mathrm{n}-1}\right] \cup \bar{K}_{n-1} \text {. }
$$

Theorem 3.17. Let $\mathrm{G}$ be a generalized quaternion group, $\mathrm{Q}_{4 n}, \mathrm{n}=\prod \mathrm{p}_{\mathrm{i}}^{\mathrm{s}_{i}}, \mathrm{~s}_{\mathrm{i}} \in \mathbb{N}$. Then

$$
\Gamma^{\operatorname{copp}}(G)= \begin{cases}K_{1}+\left[K_{1}+\left(K_{\left(p_{1}^{s_{1}+1}-2\right)} \cup n K_{2}\right) \bigcup_{i=2}^{d} K_{\left(p_{i}^{s_{i}}-1\right)}\right] \cup \bar{K}_{2\left(n-2^{s_{1}}\right)-\sum_{i=2}^{d}\left(p_{i}^{s_{i}}-1\right)}, & \text { if } p_{1}=2, \\ K_{1}+\left[\left(K_{1}+n K_{2}\right) \bigcup_{i=1}^{\mathrm{d}} K_{\left(p_{i}^{s_{i}}-1\right)}\right] \cup \bar{K}_{2(n-1)-\sum_{i=1}^{d}\left(p_{i}^{s_{i}}-1\right)^{\prime}} & \text { otherwise. }\end{cases}
$$


Proof. We consider the roup presentation. Let $A=\left\langle a^{i} \in G \mid 0 \leqslant i \leqslant 2 n-1\right\rangle$ and $B=\left\{a^{j} b \in G \mid o \leqslant j \leqslant\right.$ $2 n-1\}$ and define the sets $N_{i}=\left\{x \in A \mid O(x)=p_{i}^{s_{i}}, s_{i}>0\right\}, M=\left\{y \in B \mid O(y)=\prod p_{i}^{t}, 0 \leqslant t \leqslant s_{i}\right\}$. Then $\left|N_{i}\right|=\phi\left(p_{i}^{s_{i}}\right)=p_{i}^{s_{i}}-1$ and $|B|=2 n$. Now, we need to know $|M|$. So,

$$
|\mathrm{M}|=|\mathrm{G}|-\left\{|\boldsymbol{e}|+\left|\mathrm{N}_{\mathrm{i}}\right|+|\mathrm{B}|\right\}=2 \mathrm{n}-\left[1+\sum_{i=1}^{\mathrm{d}} \phi\left(\mathrm{p}_{\mathrm{i}}^{\mathrm{s}_{\mathrm{i}}}\right)\right] .
$$

Next we determine the cliques in both sets $A$ and $B$. In set $A$, corresponding to each prime $p_{i}$, there is a clique of size $\phi\left(p_{i}^{s_{i}}\right)$, for $1 \leqslant i \leqslant d$. Thus, set $A$ has $d$ distinct cliques. For each $a^{j} b \in B$, the centralizer $C_{G}\left(a^{j} b\right)=\left\langle a^{j} b, a^{n}\right\rangle$. Thus, the set $\left\{a^{j} b, a^{i+n} b\right\}$ for $o \leqslant j \leqslant 2 n-1$ is a clique each of size 2 , and we have $n$ such cliques. Now, for any $a^{i} b$, we have that $e \sim a^{i} b \sim Z(G)$. Therefore, the elements of $B$ make $n$ cliques each of size 2 . If $p_{1}=2$, then $\phi\left(p_{1}^{s_{1}}\right)=p_{1}^{s_{1}+1}-1$. Therefore,

$$
\begin{aligned}
\Gamma^{\mathrm{copp}}(\mathrm{G}) & =\mathrm{K}_{1}+\left[\mathrm{K}_{1}+\left(\mathrm{K}_{\left|\mathrm{N}_{1}-1\right|} \cup \mathrm{nK} \mathrm{K}_{2}\right) \bigcup_{i=2}^{\mathrm{d}} \mathrm{K}_{\left|\mathrm{N}_{i}\right|}\right] \cup \overline{\mathrm{K}}_{|\mathrm{M}|} \\
& =\mathrm{K}_{1}+\left[\mathrm{K}_{1}+\left(\mathrm{K}_{\left(\mathrm{p}_{1}^{s_{1}+1}-2\right)} \cup n \mathrm{~K}_{2}\right) \bigcup_{i=2}^{\mathrm{d}} \mathrm{K}_{\left(\mathrm{p}_{i}^{s_{i}}-1\right)}\right] \cup \bar{K}_{2\left(n-2^{s_{1}}\right)-\sum_{i=2}^{\mathrm{d}}\left(p_{i}^{s_{i}}-1\right)} .
\end{aligned}
$$

If $p_{i} \neq 2$, then $Z(G)$ should also be removed from $M$. So, $M=2(n-1)-\sum_{i=1}^{d}\left(p_{i}^{s_{i}}-1\right)$. Therefore,

$$
\Gamma^{\operatorname{copp}}(\mathrm{G})=\mathrm{K}_{1}+\left[\left(\mathrm{K}_{1}+n \mathrm{~K}_{2}\right) \bigcup_{i=1}^{\mathrm{d}} \mathrm{K}_{\left|\mathrm{N}_{i}\right|}\right] \cup \overline{\mathrm{K}}_{|\mathrm{M}|}=\mathrm{K}_{1}+\left[\left(\mathrm{K}_{1}+n \mathrm{~K}_{2}\right) \bigcup_{i=1}^{\mathrm{d}} \mathrm{K}_{\left(\mathrm{p}_{i}^{s_{i}}-1\right)}\right] \cup \bar{K}_{2(n-1)-\sum_{i=1}^{\mathrm{d}}\left(p_{i}^{s_{i}}-1\right)} .
$$

The connectivity, regularity, completeness, and planarity of the commuting order product prime graph on generalized quaternion groups is given in Proposition 3.18.

Proposition 3.18. Let $\mathrm{G}$ be a generalized quaternion group, $\mathrm{Q}_{4 \mathrm{n}}$. Then $\Gamma^{\mathrm{copp}}(\mathrm{G})$ is connected only if $\mathrm{n}=2^{\mathrm{s}}, \mathrm{s}>0$ and planar if $\mathrm{n}=2$, but not complete and not regular.

Proof. Suppose $n=2^{s}$, then $G$ is a p-group. Let $v_{i}, v_{j}, i \neq j$, be any two vertices of $\Gamma^{\operatorname{copp}}(\mathrm{G})$. Then $v_{i} \sim e \sim v_{j}$, for all $v_{i}, v_{j} \in \mathrm{V}\left(\Gamma^{\operatorname{copp}}(\mathrm{G})\right)$. Hence, each vertex is reachable from the others through $e$. Therefore, $\Gamma^{\operatorname{copp}}(G)$ is connected. Suppose on the other hand that $n \neq 2^{s}$, then $|G|=4 p^{s}$, and so, there exists $x \in G \ni o(x)=2 p^{s}$ which are not reachable from others. Hence, $\Gamma^{\operatorname{copp}}(\mathrm{G})$ is not connected in this case. For planarity, if $p=2$, then $|\mathrm{G}|=8$ and by Theorem 3.16, the maximum complete component is $\mathrm{K}_{4}$, which is planar. For completeness, $a^{i} b \times a^{i+1} b \neq a^{i+1} b \times a^{i} b$. Therefore, $\left\{a^{i} b, a^{i+1} b\right\}$ is an independent set. Hence, $\Gamma^{\operatorname{copp}}(\mathrm{G})$ is not complete. For regularity, let $v$ be an arbitrary vertex of $\Gamma^{\operatorname{copp}}(\mathrm{G})$, then $e \sim v$, therefore, $\operatorname{deg}(e)>\operatorname{deg}(v)$ for all non-central vertex $v$ of $\Gamma^{\operatorname{copp}}(G)$. Hence, $\Gamma^{\operatorname{copp}}(G)$ is not regular.

Various chromatic numbers of the commuting order product prime graph on generalized quaternion groups are investigated here. The investigation begins by giving the clique number of the commuting order product prime graph on generalized quaternion groups in Propositions 3.19 and 3.20.

Proposition 3.19. Let $\mathrm{G}$ be a generalized quaternion group, $\mathrm{Q}_{4 \mathrm{n}}, \mathrm{n}=\mathrm{p}^{\mathrm{s}}, \mathrm{s} \in \mathbb{N}$. Then

$$
\omega\left(\Gamma^{\operatorname{copp}}(G)\right)= \begin{cases}2 n, & \text { if } p=2 \\ 4, & \text { if } n=3 \\ n, & \text { otherwise. }\end{cases}
$$


Proof. Suppose $p=2$ and consider the group presentation. Let $A=\left\{a^{i}\right\}, B=\left\{a^{i} b, a^{i+n} b\right\}$, for $0 \leqslant i \leqslant$ $2 n-1$. Then $A \triangleleft G$, thus, $A$ is a clique of size $2 n$. Since the centralizer, $C_{G}\left(a^{i} b\right)=\left\langle a^{i} b, a^{n}\right\rangle$, then $B$ is a clique of size 2. But $|B|<|A|$, therefore, $A$ is the maximum clique and so, $\omega\left(\Gamma^{\operatorname{copp}}(G)\right)=|A|=2 n$. If $n=3$, then $|G|=12$, so we have the cliques; $\left\{e, a^{2}, a^{4}\right\}$ of size three and $\left\{e, a^{3}, a^{i} b, a^{i+n} b\right\}$ of size 4. Therefore, $\omega\left(\Gamma^{\operatorname{copp}}(\mathrm{G})\right)=4$. If $p \neq 2, n>3$, then $|\mathrm{G}|=4 \mathrm{p}^{\mathrm{s}}$. Thus, there exist some elements, $x \in A$ such that $O(x)=2 p^{s}$, meaning that the entire $A$ is not clique, but corresponding to the prime, $p$, the set $A^{\prime}=\left\{y \in A \mid O(y)=p^{t}, 0 \leqslant t \leqslant s\right\}$ is a clique, such that $\left|A^{\prime}\right|=p^{s}$. Now $\left|A^{\prime}\right|>|B|$, thus, $\omega\left(\Gamma^{\operatorname{copp}}(\mathrm{G})\right)=\left|A^{\prime}\right|=\mathrm{p}^{\mathrm{s}}=\mathrm{n}$.

Proposition 3.20. Let $\mathrm{G}$ be a generalized quaternion group, $\mathrm{Q}_{4 n}$, for $\mathrm{n}=\prod_{i=1}^{\mathrm{d}} \mathrm{p}_{i}^{s_{i}}, s_{i} \in \mathbb{N}$, for some prime $\mathrm{p}$. Then

$$
\omega\left(\Gamma^{\operatorname{copp}}(G)\right)= \begin{cases}p_{1}^{s_{1}+1}, & \text { if } p_{1}^{s_{1}+1}>\max \left(p_{i}^{s_{i}}\right), 2 \leqslant i \leqslant d, \\ \max \left(p_{i}^{s_{i}}\right), & \text { if } p_{1}^{s_{1}+1}<\max \left(p_{i}^{s_{i}}\right) \text { or if } p_{i} \neq 2 .\end{cases}
$$

Proof. Consider the presentation in Theorem 3.17. If $p_{1}=2$, then the connected component of $\Gamma^{\operatorname{copp}}(\mathrm{G})$ is the join union of $K_{p_{1}^{s_{1}+1}}, n K_{4}$ and $\bigcup_{i=2}^{d} K_{p_{i}}^{s_{i}}$. Thus, the maximum complete component is either, $K_{p_{1}^{s_{1}+1}}$ or $\max \left(K_{p_{i}^{s_{i}}}\right)$, for $2 \leqslant i \leqslant d$. Hence, $\omega\left(\Gamma^{\operatorname{copp}}(G)\right)=p_{1}^{s_{1}+1}$ if $p_{1}^{s_{1}+1}>\max \left(K_{p_{i}^{s_{i}}}\right)$ and $\omega\left(\Gamma^{\operatorname{copp}}(G)\right)=\max \left(p_{i}^{s_{i}}\right)$ if $p_{1}^{s_{1}+1}<\max \left(K_{p_{i}}^{s_{i}}\right)$. If $p_{i} \neq 2$, then the connected component of $\Gamma^{\mathrm{copp}}(\mathrm{G})$ is a join union of $n K_{4}$ and $\bigcup_{i=1}^{d} K_{p_{i}}^{s_{i}}$. Thus, the maximum complete component is $\max \left(K_{p_{i}}^{s_{i}}\right)$. Hence, $\omega\left(\Gamma^{\operatorname{copp}}(G)\right)=\max \left(p_{i}^{s_{i}}\right)$.

The vertex chromatic number of the commuting order product prime graph on generalized quaternion group is given in Propositions 3.21 and 3.22, respectively.

Proposition 3.21. Let $\mathrm{G}$ be a generalized quaternion group, $\mathrm{Q}_{4 \mathrm{n}}, \mathrm{n}=\mathrm{p}^{\mathrm{s}}, \mathrm{s} \in \mathbb{N}$. Then $\chi(\Gamma \operatorname{copp}(\mathrm{G}))=$ $\omega\left(\Gamma^{\operatorname{copp}}(\mathrm{G})\right)$.

Proof. Let $A=\left\{a^{i} \in G, 0 \leqslant i \leqslant 2 n-1\right\}, B=\left\{a^{i} b \in G, 0 \leqslant i \leqslant 2 n-1\right\}$ and $A^{\prime}=\left\{a^{i} \in A \mid O\left(a^{i}\right)=\right.$ $\left.p^{t}, 0 \leqslant t \leqslant s\right\}$. Then $|A|=|B|=2 n,\left|A^{\prime}\right|=\phi\left(p^{s}\right)=p^{s}$. Since the centralizer, $C_{G}\left(a^{i} b\right)=\left\langle a^{i} b, a^{n}\right\rangle$, then the pair $\left\{a^{i} b, a^{i+n} b\right\}$, for $0 \leqslant i \leqslant n-1$ are the cliques in $B$. Thus, set $B$ contains $n$ such cliques each of size two. Let $x$ be an arbitrary non-central element of $A$, then $a^{i} b \nsim x \nsim a^{i+1} b$. Therefore, $\left\{a^{i} b, x, a^{i+1} b\right\}$ is an independent set and so all its elements can be assigned with same color. That is the colors for the maximum component of the graph are sufficient to color all the vertices of the graph. Therefore, $\chi\left(\Gamma^{\operatorname{copp}}(\mathrm{G})\right)=\omega\left(\Gamma^{\operatorname{copp}}(\mathrm{G})\right)$.

Proposition 3.22. Let $\mathrm{G}$ be a generalized quaternion group, $\mathrm{Q}_{4 \mathrm{n}}$, for $\mathrm{n}=\prod \mathrm{p}_{i}^{s_{i}}, \mathrm{~s}_{\mathrm{i}}>0$. Then $\chi\left(\Gamma^{\operatorname{copp}}(\mathrm{G})\right)=$ $\left|\max \left(p_{i}^{s_{i}}\right)\right|$.

Proof. Since $n=\prod p_{i}^{s_{i}}$, then $|G|=4 \prod p_{i}^{s_{i}}$. Let $A_{i}=\left\{x \in G \mid O(x)=p_{i}^{s_{i}}, s_{i}>0\right\}, B=\{y \in G \mid O(y)=$ $\left.\prod p_{i}^{t}, 1 \leqslant t \leqslant s_{i}\right\}$ and $C=\left\{a^{i} b, a^{i+n} b, 0 \leqslant i \leqslant n-1\right\}$. Now sets $A, B$, and $C$ are the sets consisting of all the vertices of $\Gamma \operatorname{copp}(G)$. So, $\{x, y, c\}, \forall c \in C$ is an independent set, and all the elements of the set can therefore be assigned with same color. Thus, the colors for the vertices of the maximum complete subgraph is sufficient to properly color the vertices of the other components. But by Proposition 3.20, the maximum complete component is $\left|\max \left(p_{i}^{s_{i}}\right)\right|$. Therefore, $\chi\left(\Gamma^{\operatorname{copp}}(G)=\left|\max \left(p_{i}^{s_{i}}\right)\right|\right.$.

The dominated chromatic number of the commuting order product prime graph on generalized quaternion group is given in Propositions 3.23 and 3.24, respectively.

Proposition 3.23. Let $\mathrm{G}$ be a generalized quaternion group, $\mathrm{Q}_{4 \mathrm{n}}, \mathrm{n}=\mathrm{p}^{\mathrm{s}}, \mathrm{s} \in \mathbb{N}$. Then,

$$
\chi_{\text {dom }}\left(\Gamma^{\operatorname{copp}}(G)\right)= \begin{cases}2 \mathrm{n}, & \text { if } \mathrm{p}=2, \\ 6, & \text { if } \mathrm{n}=3, \\ 2 \mathrm{n}-1, & \text { otherwise. }\end{cases}
$$


Proof. If $\mathrm{p}=2$, then by Propositions 3.19 and 3.21, $\Gamma^{\mathrm{copp}}(\mathrm{G})$ required $2 \mathrm{n}$ distinct colors for proper vertex coloring. Assume that each color corresponds to a color class. So, we need to check if each of these colors is dominated by some vertices of the graph. Pick arbitrary $v \in \mathrm{V}\left(\Gamma^{\mathrm{copp}}(\mathrm{G})\right)$, then $e \sim v \sim \mathrm{Z}(\mathrm{G})$. Thus, e and $Z(G)=a^{n}$ are central vertices. Hence, the central vertices $e$ and $a^{n}$ dominate all the color classes of the vertices of the graph. Therefore, the color classes are; $C_{1}=\{e\}, C_{2}=\left\{a^{n}\right\}, C_{3}=\left\{a, a b, a^{2} b, \ldots, a^{n-1} b\right\}$, $C_{4}=\left\{a^{2}, a^{n} b, a^{n+1} b, \ldots, a^{2 n-1} b\right\}, C_{5}=\left\{a^{3}\right\}, C_{6}=\left\{a^{4}\right\}, \ldots, C_{2 n}=\left\{a^{2 n-1}\right\}$. These color classes are all dominated by $e$ and $a^{n}$ and vice versa. Thus, $\chi_{\operatorname{dom}}\left(\Gamma^{\operatorname{copp}}(\mathrm{G})\right)=2 \mathrm{n}$. If $n=3$, then $|\mathrm{G}|=12$. Thus, there exist two isolated vertices which cannot be dominated by any other vertex, which must be assigned with distinct color classes. By Propositions 3.19 and 3.21, four colors are sufficient to properly color the vertices of the graph. Therefore, $\chi_{\operatorname{dom}}\left(\Gamma^{\operatorname{copp}}(G)\right)=6$. If $p \neq 2, n>3$, then by Proposition $3.18, \Gamma^{\operatorname{copp}}(G)$ is disconnected, therefore has some isolated vertices, which cannot be dominated by any other vertex of the graph and so each of them must have distinct color class. So, the dominated chromatic number will be the sum of the vertex chromatic number and the number of the isolated vertices of the graph. Therefore, the color classes are; $C_{1}=\{e\}, C_{2}=\left\{a^{n}\right\}, C_{3}=\left\{a^{2}, b, a b, \ldots, a^{n-1} b\right\}, C_{4}=\left\{a^{4}, a^{n} b, a^{n+1} b, \ldots, a^{2 n-1} b\right\}, C_{5}=$ $\left\{a^{6}\right\}, C_{6}=\left\{a^{8}\right\}, \ldots, C_{n}=\left\{a^{2 n-2}\right\}, C_{n+1}=\{a\}, C_{n+2}=\left\{a^{3}\right\}, C_{n+3}=\left\{a^{5}\right\}, \ldots, C_{2 n-1}=\left\{a^{2 n-1}\right\}$. Thus, $\chi_{\text {dom }}\left(\Gamma^{\operatorname{copp}}(\mathrm{G})\right)=2 \mathrm{n}-1$.

Proposition 3.24. Let $\mathrm{G}$ be a generalized quaternion group, $\mathrm{Q}_{4 n}$, where $\mathrm{n}=\prod \mathrm{p}_{i}^{s_{i}}, \mathrm{~s}_{\mathrm{i}}>0$. Then

$$
\chi_{\operatorname{dom}}\left(\Gamma^{\operatorname{copp}}(G)\right)= \begin{cases}2 n-\sum_{i=2}^{d}\left(p_{i}^{s_{i}}-1\right), & \text { if } p_{1}=2, s>0, \\ \left|\max \left(p_{i}^{s_{i}}\right)\right|+2(n-1)-\sum_{i=1}^{d}\left(p_{i}^{s_{i}}\right), & \text { otherwise, }\end{cases}
$$

where $\mathrm{d}$ is the number of prime divisors of $\mathrm{n}$.

Proof. If $n=\prod p_{i}^{s_{i}}$, then $|G|=4 \prod p_{i}^{s_{i}}$ and so there exists a set $I=\left\{h \in G \mid O(h)=\prod p_{i}^{t}, t>0\right\}$. By Propositions 3.20 and 3.22, there is need for $\left|\max \left(p_{i}^{s_{i}}\right)\right|$ colors to properly color the vertices of $\Gamma^{\operatorname{copp}}(G)$. Hence, each color corresponds to distinct color class. Since set I contains some isolated vertices, which cannot be dominated by any other vertex, then each of these vertices must be assigned with distinct color classes. Therefore, for proper dominated coloring, we need $\left|\max \left(p_{i}^{s_{i}}\right)\right|+|I|$ color classes. Thus, $\chi_{\text {dom }}\left(\Gamma^{\operatorname{copp}}(G)\right)=\left|\max \left(p_{i}^{s_{i}}\right)\right|+|I|$. If $p_{1}=2$, then by Theorem 3.17, $|I|=2\left(n-2^{s_{1}}\right)-\sum_{i=2}^{d}\left(p_{i}^{s_{i}}-1\right)$. Therefore, $\chi_{\operatorname{dom}}\left(\Gamma^{\operatorname{copp}}(G)\right)=\left|\max \left(p_{i}^{s_{i}}\right)\right|+2\left(n-2^{s_{1}}\right)-\sum_{i=2}^{d}\left(p_{i}^{s_{i}}-1\right)=2 n-\sum_{i=2}^{d}\left(p_{i}^{s_{i}}-1\right)$, since $\left|\max \left(p_{i}^{s_{i}}\right)\right|=$ $2^{s_{1}+1}$ if $p_{1}=2$. If $p_{i} \neq 2$, then still by Theorem $3.17,|I|=2(n-1)-\sum_{i=1}^{d}\left(p_{i}^{s_{i}}-1\right)$. Therefore, $\chi_{\text {dom }}\left(\Gamma^{\operatorname{copp}}(G)\right)=\left|\max \left(p_{i}^{s_{i}}\right)\right|+2(n-1)-\sum_{i=1}^{d}\left(p_{i}^{s_{i}}-1\right)$.

The locating chromatic number is distance based, so can only be obtained in a connected graph. As a result, we consider only the situation when $n=2^{s}, s \in \mathbb{N}$. The result on the locating chromatic number is given in Proposition 3.25.

Proposition 3.25. Let $\mathrm{G}$ be a generalized quaternion group, $\mathrm{Q}_{2^{\alpha}}, \alpha \in \mathbb{N}$. Then, $\chi_{\mathrm{L}}\left(\Gamma^{\mathrm{copp}}(\mathrm{G})\right)=2 \mathrm{n}+1$.

Proof. Define the distance between the vertex $v$ and the color classes $C_{i}$, by $d\left(v, C_{i}\right)=\min \left\{d(v, a) \mid a \in C_{i}\right\}$. Define also the color code of the vertex $v$ as $C_{\pi}(v)=\left(d\left(v, C_{1}\right), d\left(v, C_{2}\right), \ldots, d\left(v, C_{k}\right)\right)$. Now consider the $2 n$ color classes given in Proposition 3.21 and pick arbitrary vertex $u \in V\left(\Gamma^{\operatorname{copp}}(\mathrm{G})\right)$, then $d(u, a b)=$ $d\left(u, a^{2} b\right)=\cdots=d\left(u, a^{n-1} b\right)$, also $d\left(u, a^{n} b\right)=d\left(u, a^{n+1} b\right)=\cdots=d\left(a^{2 n-1} b\right)$. Thus, $C_{\pi}(a b)=$ $C_{\pi}\left(a^{2} b\right)=\cdots=C_{\pi}\left(a^{n-1} b\right)$ and $C_{\pi}\left(a^{n} b\right)=C_{\pi}\left(a^{n+1} b\right)=\cdots=C_{\pi}\left(a^{2 n-1} b\right)$, which is problem to this coloring. That is this coloring is not proper, hence, we need to add some color classes, so that $C_{\pi}(v i) \neq$ $\mathrm{C}_{\pi}\left(v_{j}\right)$, for all $v_{i}, v_{j} \in \mathrm{V}\left(\Gamma^{\operatorname{copp}}(\mathrm{G})\right)$. Observe that $\mathrm{d}\left(\mathrm{a}^{i}, v\right) \neq \mathrm{d}\left(\mathrm{a}^{\mathrm{i}} \mathrm{b}, v\right)$, for all $v \in \mathrm{V}\left(\Gamma^{\operatorname{copp}}(\mathrm{G})\right)$, then $\left(a^{i}, a^{i} b\right)$ can be in a class. Therefore, we have the following color classes; $C_{1}=\{e\}, C_{2}=\left\{a^{\mathfrak{n}}\right\}, C_{3}=\{a, b\}$, $C_{4}=\left\{a^{2}, a b\right\}, C_{5}=\left\{a^{3}, a^{2} b\right\}, \ldots, C_{n+2}=\left\{a^{n+1}, a^{n-1} b\right\}, C_{n+3}=\left\{a^{n+2}, a^{n} b\right\}, \ldots, C_{2 n}=\left\{a^{2 n-1}, a^{2 n-3} b\right\}$, $\mathrm{C}_{2 n+1}=\left\{\mathrm{a}^{2 n-2} \mathrm{~b}, \mathrm{a}^{2 n-1} \mathrm{~b}\right\}$. Hence, $\chi_{\mathrm{L}}\left(\Gamma^{\operatorname{copp}}(\mathrm{G})\right)=2 \mathrm{n}+1$.

The edge chromatic number of the commuting order product prime graph on generalized quaternion group is given in Propositions 3.26 and 3.27, respectively. 
Proposition 3.26. Let $\mathrm{G}$ be a generalized quaternion group, $\mathrm{Q}_{4 \mathrm{n}}$, where $\mathrm{n}=\mathrm{p}^{\mathrm{s}}, \mathrm{s}>0$. Then

$$
\chi^{\prime}\left(\Gamma^{\operatorname{copp}}(\mathrm{G})\right)= \begin{cases}4 \mathrm{n}-1, & \text { if } \mathrm{p}=2, \\ 3 \mathrm{n}, & \text { otherwise. }\end{cases}
$$

Proof. If $p=2$, then by Theorem 3.16, $\Gamma^{\operatorname{copp}}(\mathrm{G})$ is a join union of $\mathrm{K}_{2 n}$ and $n K_{4}$, with $e$ and $a^{n}$ as central vertices of each component. Pick arbitrary, $u \in V\left(K_{n}\right)$ and $v \in V\left(n K_{4}\right)$ such that $u$ and $v$ are non-central vertices of $\Gamma^{\operatorname{copp}}(G)$, then $u \nsim v$, but $u \sim\left\{e, a^{n}\right\} \sim v$. Thus, the color for the edges incident to the vertices of $K_{2 n}$ cannot be shared with that of $n K_{4}$. Hence, the colors require to be fully utilized is the sum of the colors for $K_{2 n}$ and $n K_{4}$. Since $n K_{4}=K_{4} \cup K_{4} \cup \cdots \cup K_{4}$ n-tuple and two edges from each $K_{4}$ are incident to the vertex $e$, while the other two edges are incident to the other central vertex $a^{n}$, then the edge colors for $\frac{1}{2}\left(n K_{4}\right)$ are sufficient to fully color the whole $n K_{4}$. Therefore, $\chi^{\prime}(\Gamma \operatorname{copp}(G))=\chi^{\prime}\left(K_{2 n}\right)+\chi^{\prime}\left(\frac{1}{2} n K_{4}\right)=$ $(2 n-1)+2 n=4 n-1$. If $p \neq 2$, then still by Theorem 3.16, $\Gamma^{\operatorname{copp}}(\mathrm{G})$ have some isolated vertices. But these vertices are edge-less, so we do not consider them in edge coloring. Therefore, the connected component of the graph is the join union of $K_{n}$ and $n K_{4}$, whose each vertex is adjacent to the central vertices $\left\{e, a^{n}\right\}$. By using similar argument as in the previous case, we have that $\chi^{\prime}\left(\Gamma^{\operatorname{copp}}(\mathrm{G})\right)=\chi^{\prime}\left(\mathrm{K}_{n}\right)+\chi^{\prime}\left(\frac{1}{2} n K_{4}\right)=$ $n+2 n=3 n$ since $n$ is odd.

Proposition 3.27. Let $\mathrm{G}$ be a generalized quaternion group, $\mathrm{Q}_{4 n}$, where $\mathrm{n}=\prod \mathrm{p}_{i}^{s_{i}}, s_{i}>0$. Then

$$
\chi^{\prime}\left(\Gamma^{\text {copp }}(G)\right)= \begin{cases}2\left(n+2^{s_{1}}\right)-\left(1-\sum_{i=2}^{d}\left(p_{i}^{s_{i}}-1\right)\right), & \text { if } p_{1}=2, \\ 1+2 n+\sum_{i=1}^{d}\left(p_{i}^{s_{i}}\right), & \text { otherwise, }\end{cases}
$$

where $\mathrm{d}$ is the number of prime divisors of $\mathrm{n}$.

Proof. By Theorem 3.17, $\Gamma^{\operatorname{copp}}(\mathrm{G})$ has some edge-less vertices, so they will not be considered in edge coloring of the graph. Therefore, we consider the connected component of the graph, which is $\mathrm{K}_{1}+$ $\left[K_{1}+\left(K_{\left(p_{1}^{s_{1}+1}-2\right)} \cup n K_{2}\right) \cup \bigcup_{i=2}^{d} K_{\left(p_{i}^{s_{i}}-1\right)}\right]$, where $e$ is the central vertex, that is vertex of $K_{1}$ and also $a^{n}$ is the central vertex of $K_{\left(p_{1+1}^{s_{i}}-2\right)} \cup n K_{2}$. Pick $u \in V\left(K_{\left(p_{1}^{s_{1+1}}\right)-2}\right), v_{i} \in V\left(\cup_{i=2}^{d} K_{\left(p_{i}^{s_{i}}-1\right)}\right)$ and $w_{j}=V\left(n K_{2}\right)$, for $1 \leqslant j \leqslant n$. Then $\left\{u, w_{j}, v_{i}\right\}$ is an independent set and so, the edges incident to them that are not incident to $e$ can share same color. But all the vertices of the graph are adjacent to $e$, hence, $\operatorname{deg}(e)=$ $2\left(n+2^{s_{1}}\right)-\left(1-\sum_{i=2}^{d}\left(p_{i}^{s_{i}}-1\right)\right)$, which is sufficient to color the edges incident to the other vertices. Hence, $\chi^{\prime}\left(\Gamma^{\operatorname{copp}}(G)\right)=2\left(n+2^{s_{1}}\right)-\left(1-\sum_{i=2}^{d}\left(p_{i}^{s_{i}}-1\right)\right)$. If $p_{i} \neq 2$, still by Theorem 3.17 , $e$ is the central vertex and $\operatorname{deg}(e)=1+2 n+\sum_{i=1}^{d}\left(p_{i}^{s_{i}}-1\right)$. By using similar argument as in the previous case, we have that $\chi^{\prime}\left(\Gamma^{\operatorname{copp}}(\mathrm{G})\right)=1+2 n+\sum_{i=1}^{d}\left(p_{i}^{s_{i}}-1\right)$.

The dominated and edge coloring of the commuting order product prime graph on generalize quaternion group of order twelve is given in Figure 3.

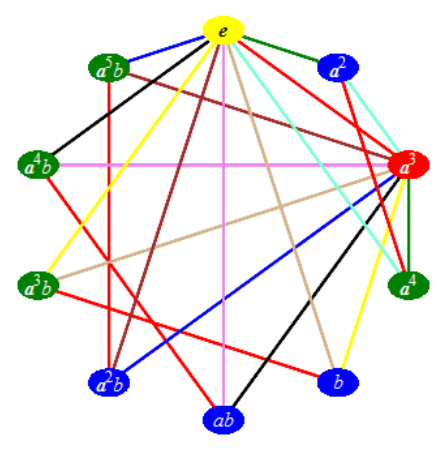

Figure 3: The dominated and edge coloring of the commuting order product prime graph of the generalized quaternion group of order twelve. 
The result on the commuting order product prime graph on quasi-dihedral group is given here. Beginning with the general structure of the graph, which is given in Theorem 3.28.

Theorem 3.28. Let $\mathrm{G}$ be a quasi-dihedral group, $\mathrm{Q}_{\mathrm{D}_{2^{n}}}$, where $\mathrm{n}>2$. Then $\Gamma^{\mathrm{copp}}(\mathrm{G})=2 \mathrm{~K}_{1}+\left(\mathrm{K}_{2\left(2^{\mathrm{n}-2}-1\right)} \cup\right.$ $2^{n-2} K_{2}$ ).

Proof. Since $G$ is a p-group, then all the elements of $G$ satisfy the first condition to be adjacent to each other. It remains to show the second condition, which is commutativity. Consider the group presentation and let $A=\langle a\rangle, B_{i}=\left\{a^{j} b, a^{j+2^{n-2}} b\right\}$, for $1 \leqslant i \leqslant 2^{n-2}, 0 \leqslant j \leqslant\left(2^{n-2}-1\right)$. Then $A$ is a clique of size $2^{n-1}$ and each $B_{i}$ is a clique of size 2 . So, we have $2^{n-2}, B_{i}$, each of size 2 . Pick arbitrary $a \in A$, $a^{j} b \in B_{i}$, then $a^{j} b \sim\left\{e, a^{2^{n-2}}\right\} \sim a$, where $a^{2^{n-2}}$ is the center of G. But $\left\{e, a^{2^{n-2}}\right\} \in A$, therefore $\left|A /\left\{e, a^{2^{n-2}}\right\}\right|=2\left(2^{n-2}-1\right)$. Thus,

$$
\Gamma^{\operatorname{copp}}(G)=\left|\left\{e, a^{2^{n-2}}\right\}\right| K_{1}+\left(K_{\mid A} /\left\{e, a^{\left.2^{n-2}\right\} \mid} \cup n\left(B_{i}\right) K_{\left|B_{i}\right|}\right)=2 K_{1}+\left(K_{2\left(2^{n-2}-1\right)} \cup 2^{n-2} K_{2}\right) .\right.
$$

The connectedness, completeness, regularity, and the planarity of the commuting order product prime graph on quasi-dihedral groups is given in Proposition 3.29.

Proposition 3.29. Let $\mathrm{G}$ be a quasi-dihedral group, $\mathrm{Q}_{\mathrm{D}_{2 n}}$, where $\mathrm{n}>2$. Then $\Gamma^{\mathrm{copp}}(\mathrm{G})$ is connected, not complete, not regular, but planar when $\mathrm{n}=3$.

Proof. Let $v_{i}, v_{j}, i \neq j$ be arbitrary non-central vertices of $\Gamma^{\text {copp }}(G)$. Then $v_{i} \sim e \sim v_{j}$, for all $v_{i}, v_{j} \in$ $\mathrm{V}\left(\Gamma^{\operatorname{copp}}(\mathrm{G})\right)$. Thus, each vertex of the graph is reachable through e. Therefore, $\Gamma^{\operatorname{copp}}(\mathrm{G})$ is connected. For completeness, by considering the group presentation, $a^{i} \nsim a^{i} b$. Thus, $\Gamma^{\operatorname{copp}}(G)$ is not complete. For regularity, since $e$ is a central vertex, then $\operatorname{deg}(e)>\operatorname{deg}\left(a^{i}\right)$, for any non-central vertex $a^{i}$. Thus, the degree of some vertices varies, therefore $\Gamma^{\operatorname{copp}}(\mathrm{G})$ is not regular. For planarity, by Theorem 3.28, the maximum complete subgraph is $K_{2^{n-1}}$. Thus, $\Gamma^{\operatorname{copp}}(\mathrm{G})$ is planar when $n=3$. Suppose on the other hand that $n=4$, then the maximum subgraph is $K_{8}$, which cannot be drawn in a plane without edge crossing. Hence, $\Gamma^{\operatorname{copp}}(\mathrm{G})$ is planar only when $\mathrm{n}=3$.

The investigation for the chromatic numbers of the commuting order product prime graph on quasidihedral group is given here. The investigation begins with the vertex chromatic number, which is given in Proposition 3.30.

Proposition 3.30. Let $\mathrm{G}$ be a quasi-dihedral group, $\mathrm{Q}_{\mathrm{D}_{2^{n}}}$, where $\mathrm{n}>2$. Then $\chi\left(\Gamma^{\mathrm{copp}}(\mathrm{G})\right)=2^{\mathrm{n}-1}$.

Proof. Consider the group presentation and let $A=\langle a\rangle, B=\left\{a^{i} b \mid 0 \leqslant i \leqslant\left(2^{n-1}-1\right)\right\}$ and $C_{k}=$ $\left\{a^{j} b, a^{j+2^{n-2}} b\right\}$, for $0 \leqslant j \leqslant 2^{n-2}-1$ and $1 \leqslant k \leqslant 2^{n-2}$. Then $C_{k} \subset B$, now for each $x \in C_{k}$, we have that $e \sim x \sim a^{2^{n-2}}$. Therefore, $C_{k} \cup\left\{e, a^{2^{n-2}}\right\}$ is a clique of size 4 and $|A|=2^{n-1}$. Thus, $A$ is the maximum clique of the graph. Hence, we need at least $2^{n-1}$ colors to color the vertices of the graph. Since $\left\{a^{i} b, a^{i+1} b\right\}$ is an independent set, then the vertices of set $B$ can share two colors which can be picked from the colors for the vertices of $A$. Thus, the colors for the vertices of set $A$ are sufficient to color the rest of the vertices for the graph. Therefore, $\chi\left(\Gamma^{\operatorname{copp}}(G)\right)=2^{n-1}$.

The dominated chromatic number of the commuting order product prime graph on quasi-dihedral group is given in Proposition 3.31.

Proposition 3.31. Let $\mathrm{G}$ be a quasi-dihedral group, $\mathrm{Q}_{\mathrm{D}_{2^{n}}}$, where $\mathrm{n}>2$. Then $\chi_{\mathrm{dom}}\left(\Gamma^{\operatorname{copp}}(\mathrm{G})\right)=2^{\mathrm{n}-1}$. 
Proof. By Proposition 3.30, there is need for $2^{n-1}$ colors to properly color the vertices of $\Gamma^{\mathrm{copp}}(\mathrm{G})$. Hence, for dominated coloring, each color corresponds to a color class, since each color is dominated by the central vertices, $e$ and $a^{2^{n-2}}$. Therefore, we have the following color classes; $C_{1}=\{e\}, C_{2}=\left\{a^{2^{n-2}}\right\}, C_{3}=$ $\left\{a, b, a b, a^{2} b, \ldots, a^{2^{n-2}-1} b\right\}, C_{4}=\left\{a^{2}, a^{2^{n-2}} b, a^{2^{n-2}+1} b, \ldots, a^{2^{n-1}} b\right\}, C_{5}=\left\{a^{3}\right\}, \ldots, C_{2^{n-2}+1}=\left\{a^{2^{n-2}-1}\right\}$, $C_{2^{n-2}+2}=\left\{a^{2^{n-2}+1}\right\}, C_{2^{n-2}+3}=\left\{a^{2^{n-2}+2}\right\}, \ldots, C_{2^{n-1}}=\left\{a^{2^{n-1}-1}\right\}$. But when $n=3$, then $Z(G)=a^{2}$, so in this case, $C_{4}=\left\{a^{3}, a^{2} b, a^{3} b\right\}$. Therefore, $\chi_{\operatorname{dom}}\left(\Gamma^{\operatorname{copp}}(G)\right)=2^{n-1}$.

The locating chromatic number of the order product prime graph on quasi-dihedral groups is given in Proposition 3.32.

Proposition 3.32. Let $\mathrm{G}$ be a quasi-dihedral group, $\mathrm{Q}_{\mathrm{D}_{2^{n}}}$, where $\mathrm{n}>2$. Then $\mathrm{\chi}_{\mathrm{L}}\left(\Gamma^{\mathrm{copp}}(\mathrm{G})\right)=2^{\mathrm{n}-1}+1$.

Proof. Consider the color classes in Proposition 3.30. Define the distance between an arbitrary vertex $v \in \mathrm{V}\left(\Gamma^{\operatorname{copp}}(\mathrm{G})\right)$ and these color classes by $\mathrm{d}\left(v, \mathrm{C}_{\mathrm{i}}\right)=\min \left\{\mathrm{d}(v, \mathrm{a}) \mid \mathrm{a} \in \mathrm{C}_{i}\right\}$. Define also the color code for the vertex $v$ as $C_{\pi(v)}=\left(d\left(v, C_{1}\right), d\left(v, C_{2}\right), \ldots, d\left(v, C_{k}\right)\right), k \in \mathbb{N}$, then $d(b, v)=d(a b, v)=\cdots=$ $\mathrm{d}\left(\mathrm{a}^{2^{n-2}} \mathrm{~b}, v\right)$. Thus, $\mathrm{C}_{\pi}(\mathrm{b})=\mathrm{C}_{\pi}(\mathrm{ab})=\cdots=\mathrm{C}_{\pi}\left(\mathrm{a}^{2^{n-2}} \mathrm{~b}\right)$, which is a problem to the locating coloring of the graph. Hence, we need to re-partition the color classes, so that $C_{\pi}(u) \neq C_{\pi}(v)$, for all $u, v \in$ $\mathrm{V}\left(\Gamma^{\operatorname{copp}}(\mathrm{G})\right)$. Thus, we have the following color classes; $C_{1}=\{e\}, C_{2}=\left\{a^{2^{n-2}}\right\}, C_{3}=\{a, b\}, C_{4}=$ $\left\{a^{2}, a b\right\}, \ldots, C_{2^{n-2}+1}=\left\{a^{2^{n-2}-1}, a^{2^{n-2}-2} b\right\}, C_{2^{n-2}+2}=\left\{a^{2^{n-2}+1}, a^{2^{n-2}-1} b\right\}, \ldots, C_{2^{n-1}}=\left\{a^{2^{n-1}-1}, a^{2^{n-1}-3} b\right\}$, $C_{2^{n-1}+1}=\left\{a^{2^{n-1}-2} b, a^{2^{n-1}-1} b\right\}$, but recall that if $n=3$, then $Z(G)=a^{2}$, so in this case, $C_{4}=\left\{a^{3}, a b\right\}$. Therefore, $\chi_{\mathrm{L}}\left(\Gamma^{\operatorname{copp}}(\mathrm{G})\right)=2^{\mathrm{n}-1}+1$.

The edge chromatic number of the commuting order product prime graph on quasi-dihedral group is given in Proposition 3.33.

Proposition 3.33. Let $\mathrm{G}$ be a quasi-dihedral group, $\mathrm{Q}_{\mathrm{D}_{2} \mathrm{n}}$, where $\mathrm{n}>2$. Then $\chi^{\prime}\left(\Gamma^{\operatorname{copp}}(\mathrm{G})\right)=2^{\mathrm{n}}-1$.

Proof. Consider the group presentation and let $A=\langle a\rangle=\left\{a^{i}\right\}, B=\left\{a^{i} b\right\}$, for $0 \leqslant i \leqslant 2^{n-1}-1$. Then $A$ is a clique, thus, $\operatorname{deg}\left(a^{i}\right)=|A|-1$, for $a^{i} \in A$. Therefore, we need $|A|$ colors to properly color the edges incident to the elements of $A$. But $|A|$ is even, so we need $|A|-1=2^{n-1}-1$ colors. For the edges incident to the vertices of the other components of the graph, since $e \sim a^{i} b \sim a^{2^{n-2}}$, then there exist edges from the vertices $a^{i} b$ to both $e$ and $a^{2^{n-2}}$. But since $\left\{a^{k} b, a^{k+2^{n-2}} b\right\}$, for $0 \leqslant k \leqslant 2^{n-2}-1$ is an independent set, then the edges between the vertices $a^{i} b$ and $e$ can share same color with the edges between $a^{i} b$ and $a^{2^{n-2}}$. Therefore, we need $(|A|-1)+|B|$ colors to properly color the edges incident to all the vertices of $\Gamma^{\operatorname{copp}}(\mathrm{G})$. Thus, $\chi^{\prime}\left(\Gamma^{\operatorname{copp}}(\mathrm{G})\right)=2^{\mathrm{n}}-1$.

\section{Acknowledgment}

The first and the third authors would like to thank Federal University of Kashere (FUK) for their total support. They also like to appreciate Universiti Teknologi Malaysia (UTM) for the financial support of International Doctoral Fellowship (IDF). The second author would like to thank Universiti Teknologi Malaysia (UTM) for its support.

\section{References}

[1] S. Arumugam, J. Bagga, K. R. Chandrasekar, On Dominator Colorings in Graphs, Proc. Indian Acad. Sci. Math. Sci., 122 (2012), 561-571. 1

[2] Asmiati, I. K. S. G. Yana, L. Yulianti, On Locating Chromatic Number of Certain Barbell Graphs, Int. J. Math. Math. Sci., 2018 (2018), 5 pages. 1

[3] A. Behtoei, B. Omoomi, On the Locating Chromatic Number of Kneser Graphs, Discrete Appl. Math., 159 (2011), 2214-2221. 1

[4] A. Behtoei, B. Omoomi, On the Locating Chromatic Number of the Cartesian Product of Graphs, Ars Combin., 126 (2016), 221-235. 1 
[5] M. Bello, N. M. Mohd Ali, S. I. Isah, N. Zulkifli, The Wiener and Zagreb Indices of the Order Product Prime Graph, Int. J. Adv. Sci. Tech., 29 (2020), 896-901. 1

[6] M. Bello, N. M. Mohd Ali, N. Zulkifli, A Systematic Approach to Group Properties Using its Geometric Structure, Eur. J. Pure Appl. Math., 13 (2020), 84-95. 1, 2.3

[7] E. A. Bertram, Some applications of graph theory to finite groups, Discrete Math., 44 (1983), 31-43. 1

[8] P. Cayley, The Theory of groups: Graphical Representation, Amer. J. Math., 1 (1878), 174-176. 1

[9] G. Chartrand, D. Erwin, M. A. Henning, P. J. Slater, P. Zhang, The locating-chromatic number of a graph, Bull. Inst. Comb. Appl., 36 (2002), 89-101. 1

[10] S. Chattopadhyay, K. L. Patra, B. K. Sahoo, Vertex Connectivity of the Power Graph of a Finite Cyclic Group, Discrete Appl. Math., 266 (2019), 259-271. 1

[11] M. Furuya, N. Matsumoto, Upper Bounds on the Locating Chromatic Number of trees, Discrete Appl. Math., 257 (2019), 338-341. 1

[12] R. Gera, C. W. Rasmussen, S. Horton, Dominator Coloring of Safe Clique Partitions, Congress, 181 (2006), 19-32. 1

[13] M. Ghanem, H. Al-Ezeh, A. Dabbour, Locating Chromatic Number of Powers of Paths and Cycles, Symmetry, 11 (2019), 8 pages. 1

[14] A. V. Kelarev, S. J. Quinn, A Combinatorial property and power graphs of Groups, Contributions to general algebra, 12 (1999), 229-235. 1

[15] X. L. Ma., M. Feng, K. S. Wang, Strong Metric Dimension of the Power Graph of a Finite Group, Discrete Appl. Math., 239 (2018), 159-164. 1

[16] T. Manjula, R. Rajeswari, Dominator Coloring of Prisim Graph, Appl. Math. Sci., 9 (2015), 1889-1894. 1

[17] K. G. Mirajkar, Y. B. Priyanka, The dominator coloring on some class of graphs, Arya Bhatta J. Math. Inform., 9 (2017), 255-262. 1

[18] B. H. Newmann, A Problem of Paul Erdös on Groups, J. Austral. Math. Soc. Ser. A, 21 (1976), 467-472. 1

[19] R. Rajkumar, P. Devi, Intersection Graphs of Cyclic Subgroups of Groups, Electron. Notes Discrete Math., 53 (2016), 15-24. 1

[20] J. A. Gallian, Contemporary Abstract Algebra, Centage Learning, Boston, (2012). 2.1, 2.2

[21] D. Vijayalakshmi, R. Kalaivani, Dominator Coloring of Sun let, gear and Helm graph families, Int. J. Pure Appl. Math. Sci., 10 (2017), 71-78. 1 